\title{
Multi drug resistant tuberculosis: a challenge in the management of tuberculosis
}

\author{
Evans Amukoye \\ Center for Respiratory Diseases Research; Kenya Medical Research Institute (KEMRI), P. O. Box 54840- \\ 00200 Nairobi, Kenya; e-mail: crdr@todays.co.ke
}

\section{SUMMARY}

\begin{abstract}
Multi drug resistant tuberculosis (MDR-TB) will not usually respond to short course chemotherapy. Unless the individual infected with this bug is treated appropriately, they can continue spreading resistant strains in the community and further fuel the tuberculosis epidemic. Diagnosis requires drug sensitivity testing and the capability to do this is not widely available. Multi drug resistant tuberculosis has been reported all over Africa but the prevalence is still low. The treatment is not only expensive but also quite prolonged and compliance cannot be overemphasized. The recent outbreaks of extensive drug resistant TB further complicate the management and control of the disease. This is a perspective on challenges of managing MDR TB and its effect on the control program the information presented is gathered from published data.
\end{abstract}

[Afr J Health Sci. 2008; 15:6-13]

\section{Introduction}

Tuberculosis (TB) is one of the fastest growing epidemics. Worldwide over 1 billion people are infected with dormant TB. Nine to eleven million people have active TB predominately in Asia, Africa, and Latin America, and almost 3 million people die of TB annually, including half a million children. WHO estimates that 4.5 million people are co-infected with the human immunodeficiency virus (HIV) and TB. TB flourishes where there is poverty, malnutrition, overcrowding, and deficient health care [1]. In Kenya the notification rate of all forms of TB over the past 5 years has been increasing by an average of $14 \%$. Reported cases of Tuberculosis have risen from 10,000 in the 1980 s to over 100,000 in 2004 [2]. The increase has been driven mainly by the high prevalence of HIV in the population. It is estimated that up to $60 \%$ of TB patients are co-infected with HIV in Kenya [2]. MDR TB, which has been demonstrated to occur in indigenous Kenyans, could complicate management of TB and erode the gains made by
DOTS if its prevalence is left to grow in the country [3].

\section{Definition}

Multi-drug resistant (MDR) Tuberculosis refers to resistance in vitro to at least Isoniazide (INH) and rifampicin (RIF). Resistance to at least two drugs other than the combination of INH and RIF is known as polyresistant tuberculosis and monoresistance is the resistance due to only one anti-tuberculosis medication [4]. This definition is based on the fact that INH plus RIF is the most important combination and resistant to them will lead to failure of short course chemotherapy even though resistant to other drugs could also lead to failure. MDR TB may be due to acquired resistance, that is strain of $M$. tuberculosis that was initially sensitive to DOTS drugs, but becomes resistant to drugs within an individual in the setting of low drug levels, intermittent therapy or effective monotherapy. Primary resistance is infection with a strain in 
M. tuberculosis that is already resistant to important first-line drugs [4].

Cases of MDR TB have been documented throughout the world including in the United States, South America, Central America, Africa, Mexico, Russia and the former Soviet Union, Western Europe, Eastern Europe, the Caribbean, Asia, New Zealand and Austria. The problem has been growing over the past several years and mathematical modeling suggests that unless a concerted effort is made to stop MDR TB, it will become a major public health problem on several continents' [3-14].

\section{Causes}

Effective therapy for disease due to $M$. tuberculosis was discovered in 1943, when streptomycin (SM) was identified. It was noticed that patients with TB who initially improved on SM often failed therapy; they no longer then responded to the drug, even at higher doses. Although a single potent drug could often eradicate the majority of tubercle bacilli, natural mutants resistant to that drug survived to become the dominant strain [15]. This mutant was then transmitted to other members of the community. INH and paramino salicylic acid (PAS) were developed shortly after SM, but when used alone, they would also develop acquired resistance [16,17]. It was then recognized that combinations of antituberculous drugs could prevent the development of drug resistance and thus, treatment regimens including more than one antituberculous drug became the standard of care.

It is worth reiterating that MDR TB, a relatively new threat to health, is an entirely man-made phenomenon; antituberculous drug resistance is the result, ultimately, of poor therapeutic practices [18]. If TB-control efforts had been well organized decades ago, it is likely that the dimensions of this problem would be significantly smaller. Thus, the introduction of systematic and effective control of drugsensitive TB, through DOTS, is our best weapon against the generation of drug resistance [19].

Directly Observed Therapy, Short Course (DOTS) refers to a specific type of tuberculosiscontrol program, requiring the following elements: 1) government commitment to a National Tuberculosis Program; 2) passive case- finding in general health services by sputum smear microscopy examination of samples from suspected TB cases; 3) standardized short-course chemotherapy administered to at least all smearpositive TB cases under specified casemanagement conditions with therapy being directly observed; 4) regular uninterrupted supply of all essential antituberculous drugs; and 5) a monitoring system for program supervision and evaluation. A strategy to manage MDR TB that takes advantage of the many strengths of the DOTS strategy is known as "DOTS-Plus" $[19,20]$.

Failure of DOTS to adequately treat drugresistant TB (whether polyresistant or MDR TB) lead to generation of further resistance and ultimately the circulation of highly resistant strains of M. tuberculosis. The result of the generation of high-grade drug resistance by inadequate therapy is not only grave for the patient, but for the community at large, as drugresistant strains are spread by the same airborne, person-to-person mechanism as a susceptible strains of TB Drugs. DOTS is ineffective for curing either form of MDR TB and for preventing further spread of drug-resistant strains in the community. It is worth to note that not all re-treatment cases of tuberculosis have MDR. In Malawi of 164 culture positive, $81 \%$ (122) were fully sensitive and only 4\% (6) had MDR [21]

Factors that favor the development and spread of MDR-TB are incomplete or inadequate therapy that can select for drug-resistant mutants of $M$ tuberculosis. Prolonged infectiousness of patients due to delayed diagnosis of MDR TB and to the absence of effective therapy also allows ongoing transmission of drug-resistant strains to susceptible contacts. Patients with cavitary lesion have a tendency to have a more chronic illness and higher chance of having MDR and should be isolated especially from immuno-compromised HIV patients [7].

Patients infected with primary drugresistant TB treated with short-course chemotherapy are less likely to be cured. Patients sick with drug-resistant TB exposed to short-course chemotherapy can further acquire resistance through inadvertent monotherapy ("the amplifier effect") [22] 


\section{Kenyan situation}

Since the introduction of short course chemotherapy in Kenya in 1993, the treatment success rate has been about $80 \%$, the death rate reduced from $1 \%$ to $0.4 \%$. In $20037 \%$ of all TB treatment were on re-treatment regime [2]. Though there is usually a higher drug resistance in the re-treatment population, it may not be evidence of MDR and outcome of re-treatment may be good, as shown in a study done in Malawi [21]. A well run control program such is available in Kenya is important in reducing secondary (acquired) drug resistance. In 1995 MDR was not reported in general population but was isolated among the refugees. [3] This is no longer so as patient seeking culture services at the Kenya Medical Research Institute (KEMRI), $11.4 \%$ of the 983 specimen tested positive for MDR tuberculosis $[23,24]$. This was the first time MDR was documented in indigenous Kenyans, even though there have been sporadic reports of MDR from public and private sector. KEMRI has undertaken a survey of drug resistant Tuberculosis and the results will soon be published. In Ethiopia, our neighbour, Multidrug-resistance (MDR) TB was reported to be about $1.2 \%$ of new cases and $12 \%$ of retreatment cases [25].

\section{Situation in children}

It is difficult to diagnose MDR tuberculosis in children and this results in delay in treatment. In South Africa the median age of diagnosing tuberculosis was 4.5 years at first TB diagnosis and 6.2 years on MDR culture confirmation. Delay in starting appropriate MDR treatment after TB diagnosis was a median of 2 days if MDR TB source cases were taken into account, but 246 days if the drug susceptibility pattern of the source case was not considered, and 283 days if there was no known tuberculosis source case. Seventeen children had smear positive tuberculosis, of which 13 had cavitatory pulmonary disease. Eight children had central nervous system TB. Thirty-six children were treated for MDR tuberculosis, of whom four died. Obtaining a detailed contact history and their culture and sensitivity result is essential in reducing the delay in starting treatment. Delay of starting appropriate MDR anti-tuberculosis treatment has potentially serious consequences [26].

\section{DOTs-plus strategy}

Evidence, and consequent estimates, suggested that the MDR situation in developing Countries, especially in sub-Saharan Africa, is deteriorating rapidly [27]. Similarly, major increases in tuberculosis have been observed in the former USSR. It was estimated that some 7-8 million new cases and 2-3 million deaths were occurring annually in the world. The global targets of reaching $85 \%$ cure rates and $70 \%$ case detection among infectious cases were established by the World Health Assembly in 1991. The WHO declaration of TB as a global emergency in 1993 and the launch of the five-element DOTS strategy in 1994-1995 resulted in countries adopting DOTS in encouraging numbers. In 2000, 148 countries including all 22 highest burden countries (HBC) responsible for $80 \%$ of cases worldwide, had adopted the new DOTS strategy [27]

WHO position statement on MDR TB is

- To achieve TB control worldwide and to prevent the emergence of antituberculous drug resistance, the WHO considers implementation of sound TBcontrol based on the DOTS strategy as a top priority.

- Recognizing that MDR TB is a considerable threat to the effectiveness of DOTS in some areas of the world, WHO strongly supports pilot projects to assess the feasibility of DOTS-Plus interventions in a variety of settings, provided DOTS is in place.

- Based on the results of these pilot projects, WHO and its partners in the newly established Working Group on DOTS-Plus for MDR TB will formulate international policy recommendations on MDR TB management. [19]

\section{Effects of HIV on MDR TB}

History of previous TB treatment, and not HIV infection, was the principal factor associated with $\mathrm{TB}$, which is resistant to at least one primary anti-TB Drug. HIV co-infection can 
shorten the period from TB infection to disease leading to lengthier periods of infectiousness. In a population-based representative data on new and previously treated patients with TB collected within an international drug resistance surveillance network, Espinal et al concluded that prior but ineffective treatment is a strong predictor of drug resistance, and that HIV is not an independent risk factor for MDR-TB [28].

The association between length of treatment and drug resistance may reflect longer treatment as a result of treatment failure in patients with drug resistance; it may also reflect irregular prior treatment for $\mathrm{TB}$, leading to drug resistance. However, as the HIV epidemic progresses in a milieu of high TB prevalence, the link with drug-resistant TB warrants constant monitoring and investigation [28].

\section{Treatment of MDR TB}

Management of MDR-TB relies on prompt recognition. Traditional culture of Mycobacterium tuberculosis isolates on solid media is slow, a commercialized molecular genetic test for a limited number of target loci might be a good alternative for a drug resistance-screening test in the context of an MDR "DOTS-plus" strategy [29].

DOT-plus strategy is more crucial in the treatment of MDR TB because of the extended duration and the potential for greater side effect. Emerging data suggest that in younger patients with little co-morbidity, the occurrence of serious adverse effect is rare and does not compromise treatment outcome. Trained individuals with experience in second line drugs are preferred to supervise therapy, but administration of medication in health centers could promote transmission of nosocomial MDR TB. A number of studies have demonstrated the effectiveness of community health workers in responding to serious challenges to health. The value of directly observed therapy in the treatment of MDR TB can not be underestimated, this could include mouth check after swallowing drugs, serum check where this is available and/or examining for side effect such as bronzing of the skin with clofazimine to ensure adherence.

The foundation of treatment consists four to five drugs to which the infecting bug is (likely) susceptible. The regime should include a parenteral drug, a first line drug to which the infective strain is likely susceptible and a fluoroquinolone whenever possible. Choose bactericidal (more efficacious) to which the patient has no allergy. Tuberculosis is sometimes partially resistant to Isoniazide, in such situations it can be used at a higher dose in conjunction with pyridoxine. Isoniazide can't be used in higher doses in conjunction with pyridoxine in situations where $\mathrm{TB}$ is fully resistant to it. Adjuvant treatments that can be used include the use of cortcosteroids in patients with severe respiratory distress, central nervous system involvement or laryngeal TB to try and prevent permanent sequelae of chronic inflammation. Surgery has been recommended with good results in patients with unilateral or focal disease that has no clinical response after 3 to 6 months of specific therapy. Other indications of surgery are high-grade resistance with extensive parenchymal damage, recurrence of positive smear or culture during or after treatment. [30].

\section{Adverse drug reactions}

The adverse drug reactions depend on drugs used. The following are some of the documented adverse reaction: Nervous system- includes headache, weakness, fatigue somnolence and insomnia. More serious reaction includes seizure, peripheral neuropathy, ototoxicity, optic neuritis and psychiatric reaction. Gastrointestinal side effects -are nausea, vomiting, diarrhea, bloating, abdominal cramps and gastritis. Rarely gastric ulcer or hepatitis may occur.

Endocrine effect such as worsening of glycemic control in diabetics, changes in menstrual period and hypothyroidism may occur.

Dermatological reactions such a skin color changes, photosensitive, and dry skin are frequently observed, while anaphylaxis and Stevens-Johnson syndrome are rare but potentially lethal. Renal and electrolyte disturbances include acute renal insufficiency, nephrolithiasis, proteinuria hypokalemia and hypomagnesemia.

Other minor adverse effects include arthralgias, myalgias, cramps and candidal vaginitis. Dehydration and/or weight loss, 
associated with gastrointestinal symptoms, may occur in the early months of treatment. [31-38].

\section{Duration of treatment}

Treatment of MDR TB is long, it is recommended that parenteral medication should continue for six months and oral medicine should continue until there is 18 to 24 months of culture negative sputum (specimen). Clinical response, radiological response and degree of resistance also determine the duration of treatment. After terminating treatment routine follow up and specimen collected for culture every 6 months for 24 months [39]. The prognosis of patients treated for MDR TB has been improving as effective drugs become available and as experience is gained in treating this condition. In a study done in South Africa out of 443 patients infected with MDR TB after 5 years $44 \%$ had died, $15 \%$ had severe chronic respiratory disease, and only $33 \%$ we cured. The situation has improved with almost an $80 \%$ cure in some setting where DOTs- plus is practiced [40].

\section{Extensive drug resistance (XDR)}

Physician working in Kwazula Natal, South Africa in 2005 noticed rapid deaths among patients with co-infection of TB and HIV [41]. Gadhi et al compiled 1539 patients with tuberculosis from this area. MDR was detected in 221 of these patients of whom 53 had XDR. All of those tested for HIV (44) were HIV positive. 52 of 53 patients died with median survival of 16 days from the time of diagnosis. $85 \%$ of the isolates had similar strain. $55 \%$ of these patients had not previously been treated [42]. XDR tuberculosis is defined as MDR (Resistant Rifampicin and Isoniazid) as well as resistant to at least two of the 6 primary classes of second line drugs, one being a fluoroquonolone and the other an injectable drug [43]. Centers for Disease Control (CDC) and WHO in 2006 documented XDR in at least 17 countries. The data showed $10 \%$ of MDR were XDR. Data from USA, Latin and Kofa showed that the XDR rates range from $4 \%$ to $19 \%$ among MDR isolates [41].

Since a percentage of MDR is XDR it means that XDR must be existing in Kenya though the number may be small as MDR is not yet a major problem in this country. The cause of MDR and XDR is due to inappropriate use of first line and second line drugs and the failure to diagnosis patients leads to increased spread of the strains diseases. Therefore it is necessary to strengthen the laboratory capacity and improve adherence to taking tuberculosis drugs or otherwise face a situation in which the spread of TB will be driven by both HIV scourge and MDR/XDR. Research into new drug sneed to be speeded up.

\section{Conclusion}

The prevalence of MDR Tuberculosis is still low in Africa compared to Eastern Europe, but it is growing and should be addressed in time. Even though the price of treatment was brought down in countries such as Peru. The cost of treating MDR TB in Kenya is estimated to be 1.3 million Kenya Shilling (personal communication by NLTP manager) which is out of the reach of most of its citizen. While HIV is fueling the TB epidemic in sub- Sahara, MDR does the same in Eastern Europe. We are facing a situation in which we will have MDR, HIV mixed in a milieu of poverty, a situation we dare not imagine.

Kenya should endeavor to reduce the emergence and spread of MDR-TB by emphasizing on strict adherence to DOTs strategy. TB drugs should be administered in DOTs registered centers. Any patient with evidence of long standing disease such as demonstration of cavities on chest X-ray, should be isolated until drug sensitivity result is known, as MDR-TB is common in long standing disease. All patients with MDR-TB should be treated promptly therefore the effort the National Leprosy and Tuberculosis Program to introduce DOTS-plus is highly supported. The introduction of DOTS-plus could also remarkably reduce the cost of treating MDR-TB.

The management of XDR will remain a challenge as there is a no effective treatment and isolation for indefinite period could raise human right issues. Health workers working with patients infected with MDR/XDR or even suspect TB should have HIV tests regularly. Those found positive should opt to work in less 
risky environments. I conclude by stating that MDR/XDR tuberculosis is a growing threat in the control of tuberculosis and this is the time to act.

\section{Acknowledgement}

I acknowledge with thanks tremendous support and permission from the Director, KEMRI that has made this write-up possible.

\section{References}

1. McKenna N.A dangerous liaison: tuberculosis and HIV. World AIDS. 1992 Sep;(23): 3 p.

2. Ministry of Health, Kenya. National Leprosy and Tuberculosis Program (NLTP). Annual report. 2003.

3. Githui WA, Juma ES, van Gorkom J, Kibuga D, Odhiambo $\mathrm{J}$ and Drobniewski F. Anti-tuberculosis drug resistance surveillance in Kenya International Journal of Tuberculosis and Lung Disease. 1998; 2: 499-505.

4. Partners in Health.- Harvard Medical School, Bill \& Belinda Gates Foundation. A DOTS-Plus Hand Book, Guide to Community based treatment of MDR TB.

5. Breathnach AS, de Ruiter A, Holdsworth GMC, Bateman NT, O'Sullivan DG, Rees PJ, Snashall D, Milburn HJ, Peters BS, Watson J, Drobniewski FA, French GL. An outbreak of multi-drug-resistant tuberculosis in a London teaching hospital. Journal of Hospital Infection. 1998; 39: 111-117.

6. Agerton T, Valway S, Gore B, pozsik C, Plikaytis B, Woodley C, Onorato I. Transmission of a highly drug-resistant strain (strain W1) of Mycobacterium tuberculosis: community outbreak and nosocomial transmission via a contaminated bronchoscope. Journal of the American Medical Association. 1997; 278: 1073-1077.
Aita J, Barrera L, Reniero A, Lopez B, Biglione J, Weisburd G, Rajmil JC, largacha C, Ritacco V . Hospital transmission of multi-drug-resistant Mycobacterium tuberculosis in Rosario, Argentina. Medicina (B. Aires) 1996:56: 48-50.

8. Chacon L, Cruz JR, Tardenchilla A. Primary resistance and MDR TB in Nicaragua. International Journal of Tuberculosis and Lung Disease. 1999; 2 (11 suppl.2): S332.

9. Grandes G. Lopez-de-Munain J, Diaz T, Rullan JV. Drug-resistant tuberculosis in Puerto Rico. 1987-1990. American Review of Respiratory Diseases. 1993; 148: 6-9

10. Raviglione MC, Rieder HL, Styblo K, Khomenko AG, Esteves K, Kochi A. Tuberculosis trends in eastern Europe and the former USSR. Tubercle and Lung Disease. 1994; 75: 400-16.

11. Stepanshina VN, Panfertsev EA, Korobova OV, Shemyakin IG, Stepanshin YG, Medvedeva IM, Dorozhkova IR. Drug-resistant strains of Mycobacterium tuberculosis isolated in Russia. International Journal of Tuberculosis and Lung Disease. 1999; 3:149-152.

12. Dawson DJ, Cheat DF, Chew WK, Haverkort FC, Lumb R, Slevers, AS. Tuberculosis in Australia, 1989-1992: bacteriologically confirmed cases and drug resistance. Medical Journal of Australia. 1995; 162: 287-290.

13. Ou Z, Xi-fu W, Jie Y. Initial drug resistance to anti-tuberculosis drugs among inpatients of a provincial chest hospital in China 1980-1991. Tubercle and Lung Disease. 1996. 77 (Suppl. 2): 62.

14. Dye C, Espinal MA.Will Tuberculosis become resistant to all antibiotics. Proceedings: Biological Sciences The Royal Society . 2001; 268: 45-52.

15. Crofton J, Mitchison D. Streptomyclin resistance in pulmonary tuberculosis. British Medical Journal. 1948:2:10091015. 
Barry VC, Conalty MI, Gaffney E. Isoniazid-resistant strains of Mycobacterium tuberculosis. Lancet 1953; 1:978-9.

17. Devadatta S, Dawson JJY, Fox W, Janardhanam B, Radhakrishna S, Ramakrishnan CV, Velu S. Attack rate of tuberculosis in a 5 year period among close family contacts of tuberculous patients under domiciliary treatment with isoniazid plus PAS or isoniazid alone. Bulletin of the World Health Organization. 1970; 42:337-351

18. Iseman MD, Tailoring a time bomb. Inadvertent genetic engineering. American Review of Respiratory Diseases. 1985; 132: 735-736.

19. World Health Organization Coordination of DOTS-Plus pilot projects for the management of MDRTB. Geneva. World Health Organization.

WHO/CDS/CPC/TB/99.262. 1999

20. World Health Organization. What is DOTS? A guide to understanding the WHO recommended TB control strategy known as DOTS. Geneva: World Health Organization, WHO/CDS/CPC/TB/99.27. 1999.

21. Salaniponi FM, Nyirenda TE, Kemp JR, Squire SB, Godfrey-Faussett P, Harries AD. Characteristics, management and outcome of patients with recurrent tuberculosis. Under routine programme conditions in Malawi. International Journal of Tuberculosis and Lung Disease. 2003; 7:948-952.

22. Farmer P, Bayona J, Becerra M, Furin J, Henry C, Hiatt H, Kim JY, Mitnick C, Nardell E.The dilemma of MDR- TB in the global era. International Journal of Tuberculosis and Lung Disease. 1998; 2: $869-876$

23. Githui WA, Meme HK, Juma ES, Kinyanjui P, Karimi F, Chakaya JM, Kangangi J, Kutwa A.Isolation of Multidrug-resistance tuberculosis strains in patients from private and public health facilities in Nairobi, Kenya. International Journal of Tuberculosis and Lung Disease. 2004; 8: 837-841
24. Githui WA, Hawken MP, Juma ES, Goefrey-faussett P, Swai OB, J, Kibuga DK, Porter JD, Wilson SM, Drobniewski FA.Surveillance of drugresistance tuberculosis and molecular evaluation of transmission of resistant strains in refugee and non refugee population in north- eastern Kenya. International Journal of Tuberculosis and Lung Disease. 2000; 4:947-955

25. Abate G. Drug-resistant tuberculosis in Ethiopia: problem scenarios and recommendation. Ethiopian Medical Journal. 2002; 40:79-86.

26. Schaaf HS, Shean K, Donald PR. Culture confirmed multi-drug resistant tuberculosis: diagnostic delay, clinical features, and outcome. Archives of Disease in Childhood. 2003; 88:11061111

27. World Health Organisation. Tuberculosis Strategy \& Operations, Stop TB Department, World Health Organization.

28. Espinal MA, Laserson K, Camacho M, Fusheng Z, Kim SJ, Tlali RE, Smith I, Suarez P, Antunes ML, George AG, Martin-Casabona N, Simelane P, Weyer $\mathrm{K}$, Binkin N and Raviglione MC. World Health Organization, communicable diseases, Geneva, Switzerland.

29. Nachega JB, Chaisson RE. Tuberculosis drug resistance: a global threat. Clinical Infectious Diseases. 2003; 36 (Suppl 1):S24-30.

30. Iseman MD. A clinician's guide to tuberculosis Lippincott Williams and Wilkins, Philadelphia, 2000. 460p

31. Ormerod LP, Horsfield N. Frequency and type of reactions to antituberculosis drugs: observations in routine treatment. Tubercle and Lung Disease. 1996; 77:37-42.

32. Holdiness MR. Clinical pharmacolokinetics of the antituberculosis drugs. Clinical Pharmacokinetics. 1984; 9:511-544.

33. Zierski M, Bek E. Side effects of drug regimens used in short course chemotherapy for pulmonary 
tuberculosis. A controlled clinical study. Tubercle 1980; 61:41-49.

34. Tack KJ, Smith JA. The safety profile of ofloxacin. American Journal of Medicine. 1989; 87:78S-81S.

35. Holdiness MR. Neurological manifestations and toxicities of the antituberculosis drugs. A review. Medical Toxicology. 1987; 2: 33-51.

36. Akhtar AJ, Crompton GK, Schonell ME. Para-aminosalicylic acid as a cause of intestinal malabsorption. Tubercle. 1968; 49:328-331.

37. Bucco T. Meligrana G, De Luca V. Neurotoxic effects of cycloserine therapy in pulmonary tuberculosis of adolescents and young adults. Scandinavian Journal of Respiratory Diseases. 1970; 71: 259-265.

38. Moore VJ. A review of side effects experienced by patients taking dofazimine. Leprosy Review. 1983; 54:327-335.

39. Fraser HF, Jazayeri D, Kempton K, Mosely M, Choi S, Pachao F, Bayona J. A system for modeling medication requirements for the management of drug resistant tuberculosis in developing countries. Medinfo. 2004; 2004 (CD): 1603

40. Schaaf HS, Botha P, Beyers N, Gie RP, Vermeulen HA, Groenewald P, Coetzee GJ and Donald PR. The 5-year outcome of multidrug resistant tuberculosis patients in the Cape Province of South Africa. Tropical Medicine and International Health. 1996 1:718-722.

41. Mario C. Raviglione MD and Smith IM, MB CHB. XDR Tuberculosisimplications for global public health. The New England Journal of Medicine. 2007; 356: 656-659.

42. Gandhi NR, Moll A, Sturm AW, Pawinski R, Govender T, Lalloo U, Zeller K, Andrews $\mathrm{J}$ and Friedland $\mathrm{G}$. Extensively drug-resistant tuberculosis as a cause of death in patients coinfected with tuberculosis and HIV in a rural area of South Africa. Lancet. 2006; 368:1575-1580.
43. Gandhi NR, Moll A and Sturm AW. Extensively drug-resistant tuberculosis (XDR-TB): recommendations for prevention and control. Weekly Epidemiological Record. 2006; 81: 431432. 